\title{
Metastatic Colorectal Adenocarcinoma
}

National Cancer Institute

\section{Source}

National Cancer Institute. Metastatic Colorectal Adenocarcinoma. NCI Thesaurus. Code C161048.

Colorectal adenocarcinoma that has spread from the original site of growth to other anatomic sites. 